\title{
Effect of Intensive Fattening of Bulls Fed a High-grain Diet on Selected Health Indicators
}

\author{
E. ŠTERCOVÁ ${ }^{1}$, D. HAAS ${ }^{1}$, A. KRÁSA ${ }^{2}$, R. LEPKOVÁ ${ }^{1}$, J. ŠTERC $^{1}$ \\ ${ }^{1}$ University of Veterinary and Pharmaceutical Sciences, Brno, Czech Republic \\ 2 Mikrop Čebín, Czech Republic
}

Received August 8, 2005

Accepted March 16, 2006

\begin{abstract}
Štercová E., D. Haas, A. Krása, R. Lepková, J. Šterc: Effect of Intensive Fattening of Bulls Fed a High-grain Diet on Selected Health Indicators. Acta Vet. Brno, 2006, 75: 209-218.

The aim of the study was to investigate the effect of intensive fattening of bulls with a high-grain diet on selected health parameters. Metabolic indicators under study were compared with those in bulls receiving a traditional diet based on maize silage. The trial included $18 \mathrm{Czech}$ pied bulls that were fed a diet containing from $75.0 \%$ to $83.6 \%$ concentrate on dry matter basis. As a control, 18 Czech pied bulls were used, given total mixed ration based on maize silage. The experiment was started after weaning when the treated group averaged 114 days of age and the control group 115 days of age. Bulls were slaughtered at an average age of 473 days in the treated group and 474 days in the control group. The experiment lasted 359 days. In the fattening period, blood and rumen fluid samples were withdrawn to evaluate rumen fermentation, acid-base balance and selected biochemical indicators. After the slaughter, samples of tuber coxae cancellous tissue were collected for the bone metabolism assessment. Among indicators under study, the rumen fermentation ones were the most affected by feeding the high-grain diet. Bulls fed the high-grain diet showed $(P \leq 0.05)$ lower rumen fluid $\mathrm{pH}$ and a higher total titration activity, a higher $(P \leq 0.01)$ total level of VFA and propionate proportion, and a lower $(P \leq 0.01)$ acetate proportion than the control bulls. Counts of infusoria were also lower $(P \leq 0.01)$. Acid-base balance was not significantly influenced by feeding the high-grain diet. The high-grain diet fed bulls showed increased serum levels of AST and phosphorus, but the differences from the control group were non-significant in most cases. In $1 \mathrm{~g}$ bone ash of the samples of tuber coxae cancellous tissue in the treated group calcium content was significantly $(P \leq 0.05)$ lower than in the control group; no significant differences were observed between other indicators under investigation.
\end{abstract}

Feeding, concentrate, rumen fermentation, acid-base balance, bone metabolism

Generally, high-grain diets cause a decrease in ruminal $\mathrm{pH}$, which may lead to the development of rumen acidosis. The risk has been pointed out by many researchers such as Nocek (1997), Owens et al. (1998) and Galyean and Rivera (2002). Excessive carbohydrate intake causes the growth of $\mathrm{G}+$ bacteria, mainly lactate producing streptococci and lactobacilli (Nocek 1997; Owen s et al. 1998). A drop in ruminal pH below 6.0, due to diets with a high proportion of grain (70-95\%, on DM basis), was reported by Harmon et al. (1985), Leedle et al. (1995), Goad et al. (1998), Hristov et al. (2001) and Schoonmaker et al. (2003). Some of them observed a concurrent increase in lactate and total VFA concentrations in rumen fluid (Harmon et al. 1985; Leedle et al. 1995; Goad et al. 1998). However, Hristov et al. (2001) observed low lactate levels in steers fed $95 \%$ concentrate diet (on DM basis), probably due to a gradual adaptation of the rumen microflora. Leedle et al. (1995) and Goad et al. (1998) reported that the proportion of acetate decreases with an increasing content of concentrate in the diet, whereas proportions of propionate, butyrate and valerate increase. Goad et al. (1998) and Hris tov et al. (2001) observed decreased counts of infusoria and ammonia concentrations when feeding highconcentrate diets. 
Due to rumen acidosis, large amounts of lactic acid infiltrate into blood from the rumen, being a primary cause of metabolic acidosis (Owens et al. 1998). Harmon et al. (1985) and Goad et al. (1998) observed only a slight decrease in blood $\mathrm{pH}$, and some decrease in bicarbonate concentrations. Greater changes in blood $\mathrm{pH}$ were reported by Leedle et al. (1995), who observed a gradual development of metabolic acidosis during a period of adaptation to a cereal-based diet that finally contained $90 \%$ concentrate. On the contrary, Hers om et al. (2003) did not find any changes in blood $\mathrm{pH}$ and bicarbonate concentrations in fattening cattle during the period of adaptation to a cereal-based diet.

The most serious liver disease directly linked to feeding a high-grain diet is purulent liver inflammation. A correlation between inflammatory lesions and mucosa ulceration in the rumen and the occurrence of liver abscesses was reported by Nagaraja and Chengappa (1998) and Galyean and Rivera (2002). They claimed that the diagnosis of liver abscesses is difficult and they are usually revealed only at the slaughter. Changes in liver enzyme activity due to feeding high-grain diets are rarely reported. Lal et al. (1991) demonstrated an increase in serum AST activity in goats that were administered wheat grain intraruminally. On the contrary, Brown et al. (1999) found out that neither AST activity nor total bilirubin were affected by feeding a diet containing $90 \%$ concentrate.

Acidogenic nutrition may also impair mineral metabolism and bone metabolism. Some researches found decreased serum Ca concentrations in sheep and cattle with rumen acidosis (Patra et al. 1993; Brown et al. 2000). On the other hand, serum P concentrations tended to be increased in animals with rumen acidosis (Patra et al. 1993; Brown et al. 1999). However, for instance B rown et al. (2000) reported decreased serum P levels in fattening cattle after intraruminal administration of cereal grain. Acidogenic nutrition has a negative impact on bone metabolism; in order to compensate for metabolic acidosis, $\mathrm{H}^{+}$ions are exchanged for bone cations, particularly $\mathrm{Ca}^{2+}$. In cattle, the diagnosis of such disorders is based on chemical analyses of tuber coxae biopsies. Doubek et al. (1994) found decreased ash contents per $1 \mathrm{~g}$ fat-free dry matter (FFDM) as well as per $1 \mathrm{~cm}^{3}$ cancellous tissue in bulls with clinical osteopathies.

\section{Materials and Methods}

The trial was performed in a selected agricultural enterprise. The trial included $18 \mathrm{Czech}$-pied bulls, fed a cerealbased diet. A proportion of concentrate in the diet ranged from $75.02 \%$ to $83.57 \%$, on DM basis. The control group included 18 bulls of the same breed, fed a maize silage-based TMR (total mixed ration). The trial was launched after the weaning which took place on the same day for both the groups. Bulls were weaned at an average age of 114 days in the treated group and 115 days in the control group, an average live weight was $139.78 \mathrm{~kg}$ in the treated group and $138.56 \mathrm{~kg}$ in the control group. They were slaughtered when the treated group averaged 473 days of age and the control group 474 days of age. Animals were loose housed in pens by groups, on deep litter, and allowed to use an outdoor area. Both groups were fed once a day, feed was placed in an outdoor concrete trough. Animal/feeder trough space ratio was 1:1. Drinking water was provided with lever drinkers. Diets were formulated with the software Animal Nutrition - Cattle (Agrokonzulta Zamberk), version 6.029. Nutrient contents in feed ingredients used were determined by methods defined in the Notice No. 124/2001 Coll. NEV (netto energy for fattening) was calculated according to the NEV equations for ruminant feedstuffs according to Sommer et al. (1994). Diet compositions are listed in Table 1 and nutrient composition of the diets in Table 2.

After habituation to the experimental diet, at about 2 month intervals, blood and rumen fluid samples were collected from 10 animals in each group, randomly selected at the beginning. Blood samples were taken four times and rumen fluid samples three times within the fattening period. For the first time only blood samples were taken, then blood and rumen fluid were sampled simultaneously. Samples were withdrawn always at the same time of the day, approximately four hours postprandially. Dates of sample collection related to different diets used are listed in Table 1. Blood samples used for the examination of acid-base balance and selected biochemical parameters were collected from vena jugularis. Rumen fluid was collected via esophageal tube.

Rumen fluid $\mathrm{pH}$ was determined by electrometry, total acidity by titration. Infusoria were counted in the FuchsRosenthal chamber. Ammonia and lactate concentrations were determined by photometry using Quantum (Stangest) analyser. Lactate concentration was determined by D-Lactic acid/L- Lactic acid test (R-Biopharm AG). Total VFA (volatile fatty acids) contents and proportions of acetate, propionate, butyrate and valerate were measured by gas chromatography. Blood samples were analysed for acid-base balance parameters with the Astrup 
Table 1. Composition of the diets for the treated and control groups and days of blood and rumen fluid sampling (* - only blood samples)

\begin{tabular}{|c|c|c|c|c|c|c|}
\hline \multirow[t]{2}{*}{ Treated group } & \multirow{2}{*}{$\begin{array}{c}\text { Diet } \\
\text { after weaning }\end{array}$} & \multirow{2}{*}{$\begin{array}{c}\text { Transitional } \\
\text { diet }\end{array}$} & \multicolumn{4}{|c|}{ Experimental diets for live weight } \\
\hline & & & $300 \mathrm{~kg}$ & $350 \mathrm{~kg}$ & $450 \mathrm{~kg}$ & $550 \mathrm{~kg}$ \\
\hline Age (days) & $114-176$ & $176-194$ & $194-261$ & $261-332$ & $332-402$ & $402-472$ \\
\hline $\begin{array}{l}\text { Sampling } \\
\text { (days of age) }\end{array}$ & - & - & $220 *$ & 282 & 351 & 421 \\
\hline Concentrates $(\%)$ & $83.0 \%$ & $82.3 \%$ & $83.6 \%$ & $81.3 \%$ & $77.4 \%$ & $75.0 \%$ \\
\hline Feed & \multicolumn{6}{|c|}{ Dry matter $(\mathrm{kg})$} \\
\hline $\mathrm{ČOT} \mathrm{L}^{1}$ & 4.49 & 1.79 & - & - & - & - \\
\hline Meadow hay & 0.92 & 1.09 & 1.37 & 1.65 & 2.29 & 2.75 \\
\hline Cereals $^{2}$ & - & 2.59 & 5.61 & 5.82 & 6.47 & 6.9 \\
\hline$\overline{\mathrm{BK}} 1^{3}$ & - & 0.69 & 1.36 & 1.36 & 1.36 & 1.36 \\
\hline
\end{tabular}

\begin{tabular}{|l|c|c|c|c|c|c|}
\hline \multirow{2}{*}{ Control group } & \multirow{2}{*}{$\begin{array}{c}\text { Diet } \\
\end{array}$} & \multicolumn{5}{|c|}{ TMR for live weight } \\
\cline { 3 - 7 } & after weaning & $200 \mathrm{~kg}$ & $300 \mathrm{~kg}$ & $350 \mathrm{~kg}$ & $450 \mathrm{~kg}$ & $550 \mathrm{~kg}$ \\
\hline Age (days) & $115-180$ & $180-195$ & $195-262$ & $262-333$ & $333-403$ & $403-473$ \\
\hline $\begin{array}{l}\text { Sampling } \\
\text { (days of age) }\end{array}$ & - & - & $221 *$ & 283 & 352 & 422 \\
\hline Concentrates (\%) & $51.0 \%$ & $34.7 \%$ & $34.7 \%$ & $34.7 \%$ & $34.7 \%$ & $34.7 \%$ \\
\hline Feed & Dry matter $(\mathrm{kg})$ & & & & & - \\
\hline ČOT L 1 & 1.35 & - & - & - & 6.95 & 7.30 \\
\hline Maize silage & 2.67 & 5.17 & 5.99 & 6.52 & 2.63 & 2.76 \\
\hline Cereals & & & & & \\
\hline BK 2 & 1.01 & 1.96 & 2.27 & 2.47 & 1.01 & 1.06 \\
\hline Ground calcite & 0.39 & 0.75 & 0.86 & 0.94 & 0.07 & 0.07 \\
\hline
\end{tabular}

1) ČOT L - pelleted concentrated feed for weaned calves

2) Cereals - a mixture of crushed cereals, wheat + barley, $1: 1$

3) BK 1 - a protein concentrate for the treated group, ingredients $-45 \%$ rapeseed meal (34\% CP), $20 \%$ soybean meal (45\% CP), $10 \%$ sulphite yeast, $7 \%$ urea, $3 \% \mathrm{NaHCO}_{3}, 7.6 \%$ ground calcite, $2.5 \%$ monocalcium phosphate,

$2.9 \% \mathrm{NaCl}, 2 \%$ mineral and vitamin supplements

4) BK 2 - protein concentrate for the control group, ingredients - rapeseed

meal, cereals, forage meals, mineral and vitamin supplements, $13 \%$ urea, 56 mg. $\mathrm{kg}^{-1}$ Flavomycin

method, using the RAPIDLAB 855 automatic analyser. Acid-base balance parameters determined included blood $\mathrm{pH}$, partial pressure of $\mathrm{CO}_{2}\left(\mathrm{pCO}_{2}\right)$, standard bicarbonate $(\mathrm{SB})$ and base excess $(\mathrm{BE})$. Total bilirubin concentrations, inorganic phosphate concentrations, activities of AST (aspartate transaminase) and GMT (gamma-glutamyl transferase) in blood serum were measured with the automated analyser COBAS MIRA S. Calcium (Ca) and magnesium $(\mathrm{Mg}$ ) concentrations in blood serum were measured by atomic absorption spectrometry.

After the slaughter, 5 carcasses in each group were withdrawn samples of tuber coxae cancellous tissue with a bone trocar. These were animals that had been withdrawn blood and rumen fluid samples before. In the samples of tuber coxae cancellous tissue, contents of ash, calcium and phosphorus in $1 \mathrm{~g}$ FFDM (fat-free dry matter) and 1 $\mathrm{cm}^{3}$ of spongy substance were measured. The values received were used to calculate $\mathrm{Ca} / \mathrm{P}$ index in ash. For all the parameters under observation, arithmetic mean (x), standard deviation (SD) and coefficient of variation (V) were calculated. For the testing of significance of differences between the high-grain and control groups the Student's $t$-test was used. Statistical data were processed with Excel (Microsoft Office 97).

\section{Results}

An average daily gain from weaning to slaughter was $1.29 \mathrm{~kg}$ in the treated group and $1.21 \mathrm{~kg}$ in the control group. An average slaughter live weight was $600.94 \mathrm{~kg}$ in the treated group and $573.33 \mathrm{~kg}$ in the control group. The differences between the groups were not 
Table 2. Nutrient content of the diets for the treated and control groups in $\mathrm{kg}$ of dry matter $(\mathrm{CP}=$ crude protein, $\mathrm{NEV}=$ netto energy for fattening)

\begin{tabular}{|l|c|c|c|r|r|c|}
\hline \multirow{2}{*}{ Treated group } & \multirow{2}{*}{$\begin{array}{c}\text { Diet } \\
\text { after weaning }\end{array}$} & \multirow{2}{*}{$\begin{array}{c}\text { Transitional } \\
\text { diet }\end{array}$} & \multicolumn{5}{|c|}{ Experimental diets for live weight } \\
\cline { 4 - 7 } & 5.41 & 6.16 & $300 \mathrm{~kg}$ & $350 \mathrm{~kg}$ & $450 \mathrm{~kg}$ & $550 \mathrm{~kg}$ \\
\hline Dry matter $(\mathrm{kg})$ & 202.86 & 194.33 & 187.71 & 183.24 & 173.86 & 168.61 \\
\hline $\mathrm{CP}\left(\mathrm{g} \cdot \mathrm{kg}^{-1}\right)$ & 102.03 & 91.43 & 80.53 & 85.71 & 94.87 & 100.30 \\
\hline Fiber $\left(\mathrm{g} \cdot \mathrm{kg}^{-1}\right)$ & 18.37 & 12.60 & 9.12 & 8.84 & 8.20 & 7.85 \\
\hline $\mathrm{Ca}\left(\mathrm{g} \cdot \mathrm{kg}^{-1}\right)$ & 7.45 & 6.63 & 6.14 & 6.03 & 5.80 & 5.66 \\
\hline $\mathrm{P}\left(\mathrm{g} \cdot \mathrm{kg}^{-1}\right)$ & 3.06 & 2.76 & 2.54 & 2.46 & 2.27 & 2.17 \\
\hline $\mathrm{Mg}\left(\mathrm{g} \cdot \mathrm{kg}^{-1}\right)$ & 7.69 & 7.96 & 8.22 & 8.13 & 7.99 & 7.91 \\
\hline $\mathrm{NEV}(\mathrm{MJ} / \mathrm{kg})$ & & & & & \\
\hline
\end{tabular}

\begin{tabular}{|l|c|r|r|r|r|c|}
\hline \multirow{2}{*}{ Control group } & \multirow{2}{*}{$\begin{array}{c}\text { Diet } \\
\text { after weaning }\end{array}$} & \multicolumn{5}{|c|}{ TMR for live weight } \\
\cline { 3 - 7 } & $200 \mathrm{~kg}$ & $300 \mathrm{~kg}$ & $350 \mathrm{~kg}$ & $450 \mathrm{~kg}$ & $550 \mathrm{~kg}$ \\
\hline Dry matter $(\mathrm{kg})$ & 5.45 & 7.93 & 9.18 & 9.99 & 10.66 & 11.19 \\
\hline $\mathrm{CP}\left(\mathrm{g} \cdot \mathrm{kg}^{-1}\right)$ & 153.35 & 129.57 & 129.37 & 129.50 & 129.44 & 129.56 \\
\hline Fiber $\left(\mathrm{g} \cdot \mathrm{kg}^{-1}\right)$ & 86.23 & 120.27 & 120.27 & 120.38 & 120.28 & 120.31 \\
\hline $\mathrm{Ca}\left(\mathrm{g} \cdot \mathrm{kg}^{-1}\right)$ & 10.70 & 6.96 & 7.03 & 6.84 & 7.05 & 6.94 \\
\hline $\mathrm{P}\left(\mathrm{g} \cdot \mathrm{kg}^{-1}\right)$ & 4.81 & 3.68 & 3.68 & 3.68 & 3.68 & 3.68 \\
\hline $\mathrm{Mg}\left(\mathrm{g} \cdot \mathrm{kg}^{-1}\right)$ & 2.77 & 1.94 & 1.94 & 1.94 & 1.94 & 1.94 \\
\hline $\mathrm{NEV}(\mathrm{MJ} / \mathrm{kg})$ & 7.38 & 7.08 & 7.08 & 7.08 & 7.08 & 7.08 \\
\hline
\end{tabular}

statistically significant. During the experiment, there was no incidence of clinical signs of any disease.

Results of rumen fluid analyses are listed in Table 3. The treated bulls showed lower rumen fluid $\mathrm{pH}$ and higher total titration acidity. The differences between the groups were significant $(P \leq 0.05)$, and in the last collection samples they were highly significant $(P \leq 0.01)$. In the high-grain diet group, infusorial counts were highly significantly $(P \leq 0.01)$ lower with the first and last sampling than in the control group. Ammonia and lactate concentrations in rumen fluid were highly significantly $(P \leq 0.01)$ higher in the high-grain diet group than in the control group with the first sampling, with other samplings the differences between the groups were not significant. In the high-grain diet group, total VFA concentrations were significantly $(P \leq 0.05)$ higher than in the control group with the first sampling, with the other two samplings they were highly significantly higher $(P \leq 0.01)$. As to percentages of different VFAs, the high-grain diet group showed highly significantly higher $(P \leq 0.01)$ proportions of propionate and valerate and highly significantly $(P \leq 0.01)$ lower proportion of acetate, and with the last two samplings also of butyrate.

Table 4 shows results of the analysis of acid-base balance parameters and selected biochemical parameters in the serum. Mean $\mathrm{pH}$ value of blood was decreased in both the groups with all the samplings and $\mathrm{pCO}_{2}$ value was increased, when compared with the reference values (Jagoš and B ouda 1981). However, except for the last sampling, the differences between the groups were not statistically significant. With the last sampling, the high-grain diet group showed highly significantly $(P \leq 0.01)$ higher blood $\mathrm{pH}$ and lower $\mathrm{pCO}_{2}$ than the control one. There were no significant differences found in SB and $\mathrm{BE}$, except for the third sampling when the high-grain diet group showed significantly $(P \leq 0.05)$ higher values of both SB and BE. There were no significant differences in serum bilirubin concentrations found between the groups. AST activity was found increased in all the samples withdrawn, but differences between the groups were non-significant, only 
Table 3. Results of rumen fluid analyses

\begin{tabular}{|c|c|c|c|c|c|c|c|c|}
\hline \multirow{2}{*}{\multicolumn{2}{|c|}{ Indicators Sample }} & \multicolumn{3}{|c|}{$\begin{array}{c}\text { Treated group } \\
n=10\end{array}$} & \multicolumn{3}{|c|}{$\begin{array}{l}\text { Control group } \\
n n=10\end{array}$} & \multirow{2}{*}{$\begin{array}{c}t \text {-test } \\
P\end{array}$} \\
\hline & & $\mathrm{x}$ & $\mathrm{sd}$ & $\mathrm{V}(\%)$ & $\mathrm{x}$ & $\mathrm{sd}$ & $\mathrm{V}(\%)$ & \\
\hline \multirow[t]{3}{*}{$\mathrm{pH}$} & $1^{\text {st }}$ & 6.469 & 0.179 & 2.764 & 6.745 & 0.295 & 4.380 & $0.0211 *$ \\
\hline & $2^{\text {nd }}$ & 6.249 & 0.361 & 5.785 & 6.612 & 0.298 & 4.513 & $0.0298 *$ \\
\hline & $3^{\text {rd }}$ & 6.193 & 0.113 & 1.832 & 6.590 & 0.267 & 4.053 & $0.0018 * *$ \\
\hline \multirow{3}{*}{$\begin{array}{l}\text { Total acidity } \\
\text { (arb.unit) }\end{array}$} & & 28.060 & 3.937 & 14.032 & 22.620 & 5.364 & 23.715 & $0.0187 *$ \\
\hline & $2^{\text {nd }}$ & 27.420 & 8.244 & 30.065 & 19.956 & 3.747 & 18.775 & $0.0229 *$ \\
\hline & $3^{\text {rd }}$ & 27.540 & 2.451 & 8.901 & 21.000 & 3.923 & 18.681 & $0.0004 * *$ \\
\hline \multirow{3}{*}{$\begin{array}{l}\text { Infusoria } \\
\text { (thousand.ml-1) }\end{array}$} & & 279.400 & 41.226 & 14.755 & 566.400 & 153.97 & 27.183 & $0.0002 * *$ \\
\hline & $2^{\text {nd }}$ & 700.000 & 259.43 & 37.061 & 690.667 & 117.85 & 17.063 & 0.9199 \\
\hline & $3^{\text {rd }}$ & 360.800 & 138.21 & 38.308 & 609.000 & 180.77 & 29.684 & $0.0035 * *$ \\
\hline \multirow{3}{*}{$\begin{array}{l}\text { Ammonia } \\
\left(\mathrm{mmol} \cdot \mathrm{l}^{-1}\right)\end{array}$} & & 26.850 & 9.530 & 35.492 & 7.600 & 3.098 & 40.759 & $8.10^{-5} * *$ \\
\hline & $2^{\text {nd }}$ & 7.020 & 2.973 & 42.352 & 7.300 & 3.091 & 42.338 & 0.8429 \\
\hline & $3^{\text {rd }}$ & 7.780 & 2.163 & 27.805 & 6.775 & 2.438 & 35.987 & 0.3542 \\
\hline \multirow{3}{*}{$\begin{array}{l}\text { Lactate } \\
\left(\mathrm{mmol} \cdot \mathrm{l}^{-1}\right)\end{array}$} & $1^{\text {st }}$ & 0.321 & 0.134 & 41.859 & 0.042 & 0.024 & 58.105 & $9.10^{-5} * *$ \\
\hline & $2^{\text {nd }}$ & 0.120 & 0.061 & 51.220 & 0.139 & 0.070 & 50.457 & 0.5395 \\
\hline & $3^{\text {rd }}$ & 0.043 & 0.029 & 66.730 & 0.073 & 0.047 & 64.185 & 0.1102 \\
\hline \multirow{3}{*}{$\begin{array}{l}\text { Total VFA } \\
\left(\mathrm{mmol} . \mathrm{l}^{-1}\right)\end{array}$} & $1^{\text {st }}$ & 132.500 & 21.268 & 16.052 & 112.200 & 21.467 & 19.133 & $0.0478 *$ \\
\hline & $2^{\text {nd }}$ & 155.620 & 23.799 & 15.293 & 126.244 & 9.407 & 7.451 & $0.0036 * *$ \\
\hline & $3^{\text {rd }}$ & 143.550 & 13.120 & 9.140 & 121.288 & 19.501 & 16.079 & $0.0090 * *$ \\
\hline \multirow{3}{*}{$\begin{array}{l}\text { Acetate } \\
(\mathrm{mol} \%)\end{array}$} & $1^{\text {st }}$ & 53.640 & 2.191 & 4.085 & 63.310 & 2.295 & 3.624 & $2.10^{-8} * *$ \\
\hline & $2^{\text {nd }}$ & 52.330 & 1.553 & 2.967 & 63.011 & 2.184 & 3.466 & $6.10^{-10} * *$ \\
\hline & $3^{\text {rd }}$ & 57.630 & 1.778 & 3.086 & 64.625 & 1.697 & 2.626 & $1.10^{-7} * *$ \\
\hline \multirow{3}{*}{\begin{tabular}{|l} 
Propionate \\
$(\mathrm{mol} \%)$
\end{tabular}} & $1^{\text {st }}$ & 30.770 & 2.990 & 9.717 & 21.880 & 3.564 & 16.290 & $1.10^{-5} * *$ \\
\hline & $2^{\text {nd }}$ & 34.700 & 3.700 & 10.662 & 15.589 & 2.646 & 16.973 & $4.10^{-10} * *$ \\
\hline & $3^{\text {rd }}$ & 26.330 & 3.517 & 13.356 & 14.625 & 1.894 & 12.950 & $9.10^{-8} * *$ \\
\hline \multirow{3}{*}{$\begin{array}{l}\text { Butyrate } \\
(\mathrm{mol} \%)\end{array}$} & $1^{\text {st }}$ & 12.790 & 1.965 & 15.363 & 12.890 & 2.976 & 23.085 & 0.9303 \\
\hline & $2^{\text {nd }}$ & 10.370 & 3.210 & 30.955 & 19.433 & 2.391 & 12.304 & $2.10^{-6} * *$ \\
\hline & $3^{\text {rd }}$ & 13.390 & 2.193 & 16.379 & 18.800 & 1.173 & 6.237 & $5.10^{-6} * *$ \\
\hline \multirow{3}{*}{$\begin{array}{l}\begin{array}{l}\text { Valerate } \\
(\mathrm{mol} \%)\end{array} \\
\end{array}$} & $1^{\text {st }}$ & 2.790 & 0.398 & 14.282 & 1.940 & 0.347 & 17.889 & $7.10^{-5} * *$ \\
\hline & $2^{\text {nd }}$ & 2.670 & 0.736 & 27.582 & 1.944 & 0.361 & 18.563 & $0.0159 *$ \\
\hline & $3^{\text {rd }}$ & 2.640 & 0.574 & 21.738 & 1.938 & 0.187 & 9.634 & $0.0037 * *$ \\
\hline
\end{tabular}

$\mathrm{x}=$ arithmetic mean, $\mathrm{sd}=$ standard deviation, $\mathrm{V}=$ coefficient of variation

$* P \leq 0.05, * * P \leq 0.01$ - significant difference between the treated and control group

with the last sampling the high-grain group showed a significantly $(P \leq 0.05)$ higher mean value. There were no significant differences found in GMT activity between the groups. Neither in Ca nor in Mg serum concentrations significant differences were found between the groups. Only with the first sampling the high-grain diet group showed a significantly $(P \leq 0.05)$ higher $\mathrm{Mg}$ concentration. Serum $\mathrm{P}$ concentrations were higher in the treated group with all the samplings, but only with the second sampling this value was highly significantly $(P \leq 0.01)$ higher than in the control group.

Results of tuber coxae cancellous tissue examination after the slaughter are given in Table 5. The treated bulls showed lower $\mathrm{Ca}$ and $\mathrm{P}$ levels in $1 \mathrm{~g}$ FFDM than the control ones, as well as lower levels of $\mathrm{Ca}$ and $\mathrm{P}$ per $1 \mathrm{~g}$ ash in the tuber coxae cancellous tissue. Differences between the groups were not significant, only the Ca level per $1 \mathrm{~g}$ ash was significantly $(P \leq 0.05)$ lower in the treated group. 
Table 4 . Results of acid-base balance and biochemical parameters

\begin{tabular}{|c|c|c|c|c|c|c|c|c|}
\hline \multirow[t]{3}{*}{ Indicators } & \multirow[t]{3}{*}{ Sample } & \multicolumn{3}{|c|}{ Treated group } & \multicolumn{3}{|c|}{ Control group } & \multirow{3}{*}{$\frac{t \text {-test }}{P}$} \\
\hline & & \multicolumn{3}{|c|}{$\mathrm{n}=10$} & \multicolumn{3}{|c|}{$\mathrm{n}=10$} & \\
\hline & & $\mathrm{x}$ & sd & $\mathrm{V}(\%)$ & $\mathrm{x}$ & $\mathrm{sd}$ & $\mathrm{V}(\%)$ & \\
\hline \multirow[t]{4}{*}{$\mathrm{pH}$} & $1^{\text {st }}$ & 7.357 & 0.031 & 0.420 & 7.339 & 0.035 & 0.476 & 0.2356 \\
\hline & $2^{\text {nd }}$ & 7.333 & 0.041 & 0.561 & 7.348 & 0.029 & 0.401 & 0.3576 \\
\hline & $3^{\text {rd }}$ & 7.370 & 0.025 & 0.346 & 7.342 & 0.050 & 0.681 & 0.1308 \\
\hline & $4^{\text {th }}$ & 7.325 & 0.057 & 0.783 & 7.254 & 0.016 & 0.225 & $0.0033 * *$ \\
\hline \multirow{4}{*}{$\begin{array}{l}\mathrm{pCO}_{2} \\
(\mathrm{kPa})\end{array}$} & $1^{\text {st }}$ & 7.647 & 0.574 & 7.508 & 7.894 & 0.883 & 11.189 & 0.4680 \\
\hline & $2^{\text {nd }}$ & 8.693 & 1.091 & 12.548 & 8.103 & 0.536 & 6.615 & 0.1490 \\
\hline & $3^{\text {rd }}$ & 7.408 & 0.871 & 11.762 & 7.592 & 0.937 & 12.337 & 0.6630 \\
\hline & $4^{\text {th }}$ & 7.442 & 1.100 & 14.779 & 9.056 & 0.432 & 4.771 & $0.0011 * *$ \\
\hline \multirow{4}{*}{$\begin{array}{l}\text { SB } \\
\left(\mathrm{mmol} \cdot \mathrm{l}^{-1}\right)\end{array}$} & $1^{\text {st }}$ & 27.200 & 0.858 & 3.153 & 26.560 & 1.175 & 4.424 & 0.1811 \\
\hline & $2^{\text {nd }}$ & 28.630 & 1.450 & 5.064 & 28.220 & 2.092 & 7.412 & 0.6166 \\
\hline & $3^{\text {rd }}$ & 27.760 & 0.963 & 3.468 & 26.267 & 1.503 & 5.723 & $0.0184 *$ \\
\hline & $4^{\text {th }}$ & 24.870 & 1.397 & 5.617 & 24.138 & 1.165 & 4.827 & 0.2344 \\
\hline \multirow{4}{*}{$\begin{array}{l}\text { BE } \\
\left(\mathrm{mmol.} \mathrm{l}^{-1}\right)\end{array}$} & $1^{\text {st }}$ & 4.450 & 0.897 & 20.162 & 3.700 & 1.315 & 35.537 & 0.1536 \\
\hline & $2^{\text {nd }}$ & 5.800 & 1.444 & 24.906 & 5.440 & 2.247 & 41.306 & 0.6750 \\
\hline & $3^{\text {rd }}$ & 4.860 & 1.322 & 27.197 & 3.189 & 1.688 & 52.950 & $0.0272 *$ \\
\hline & $4^{\text {th }}$ & 1.390 & 1.602 & 115.23 & 0.700 & 1.384 & 197.69 & 0.3318 \\
\hline \multirow{4}{*}{$\begin{array}{l}\text { Bilirubin } \\
\left(\mu \mathrm{mol} .1^{-1}\right)\end{array}$} & $1^{\text {st }}$ & 6.420 & 1.696 & 26.422 & 7.080 & 2.063 & 29.135 & 0.4447 \\
\hline & $2^{\text {nd }}$ & 3.790 & 0.649 & 17.120 & 4.250 & 0.936 & 22.024 & 0.2177 \\
\hline & $3^{\text {rd }}$ & 4.030 & 0.302 & 7.494 & 4.733 & 0.916 & 19.363 & 0.0539 \\
\hline & $4^{\text {th }}$ & 5.010 & 0.818 & 16.337 & 5.350 & 0.638 & 11.932 & 0.3308 \\
\hline \multirow{4}{*}{ 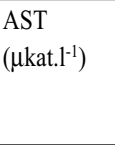 } & $1^{\text {st }}$ & 1.859 & 0.366 & 19.713 & 1.622 & 0.292 & 18.016 & 0.1272 \\
\hline & $2^{\text {nd }}$ & 2.029 & 0.683 & 33.653 & 1.636 & 0.225 & 13.735 & 0.1119 \\
\hline & $3^{\text {rd }}$ & 1.913 & 0.289 & 15.110 & 1.739 & 0.253 & 14.584 & 0.1830 \\
\hline & $4^{\text {th }}$ & 1.901 & 0.255 & 13.410 & 1.699 & 0.139 & 8.201 & $0.0500 *$ \\
\hline \multirow{4}{*}{$\begin{array}{l}\text { GMT } \\
\left(\mu \text { kat. } l^{-1}\right)\end{array}$} & $1^{\text {st }}$ & 0.391 & 0.115 & 29.395 & 0.393 & 0.068 & 17.301 & 0.9627 \\
\hline & $2^{\text {nd }}$ & 0.432 & 0.124 & 28.805 & 0.387 & 0.108 & 27.938 & 0.3994 \\
\hline & $3^{\text {rd }}$ & 0.408 & 0.115 & 28.273 & 0.421 & 0.093 & 22.100 & 0.7900 \\
\hline & $4^{\text {th }}$ & 0.530 & 0.058 & 10.930 & 0.586 & 0.073 & 12.533 & 0.0799 \\
\hline \multirow{4}{*}{$\begin{array}{l}\mathrm{Ca} \\
\left(\mathrm{mmol} \cdot \mathrm{l}^{-1}\right)\end{array}$} & $1^{\text {st }}$ & 2.330 & 0.071 & 3.055 & 2.318 & 0.061 & 2.634 & 0.6905 \\
\hline & $2^{\text {nd }}$ & 2.682 & 0.071 & 2.653 & 2.579 & 0.151 & 5.865 & 0.0736 \\
\hline & $3^{\text {rd }}$ & 2.401 & 0.088 & 3.686 & 2.467 & 0.110 & 4.459 & 0.1678 \\
\hline & $4^{\text {th }}$ & 2.486 & 0.186 & 7.468 & 2.333 & 0.151 & 6.472 & 0.0664 \\
\hline \multirow{4}{*}{$\begin{array}{l}\mathrm{P} \\
\left(\mathrm{mmol} \cdot \mathrm{l}^{-1}\right)\end{array}$} & $1^{\text {st }}$ & 2.295 & 0.174 & 7.590 & 2.154 & 0.197 & 9.166 & 0.1076 \\
\hline & $2^{\text {nd }}$ & 2.655 & 0.200 & 7.527 & 2.336 & 0.161 & 6.882 & $0.0010 * *$ \\
\hline & $3^{\text {rd }}$ & 2.348 & 0.133 & 5.664 & 2.233 & 0.146 & 6.523 & 0.0906 \\
\hline & $4^{\text {th }}$ & 2.793 & 0.312 & 11.183 & 2.784 & 0.172 & 6.188 & 0.9385 \\
\hline \multirow{4}{*}{$\begin{array}{l}\mathrm{Mg} \\
\left(\mathrm{mmol} .1^{-1}\right)\end{array}$} & $1^{\text {st }}$ & 0.964 & 0.030 & 3.139 & 0.893 & 0.090 & 10.127 & $0.0382 *$ \\
\hline & $2^{\text {nd }}$ & 0.938 & 0.043 & 4.628 & 0.919 & 0.056 & 6.079 & 0.4069 \\
\hline & $3^{\text {rd }}$ & 1.005 & 0.052 & 5.207 & 1.006 & 0.053 & 5.265 & 0.9819 \\
\hline & $4^{\text {th }}$ & 0.979 & 0.062 & 6.360 & 1.018 & 0.045 & 4.416 & 0.1441 \\
\hline
\end{tabular}

$\mathrm{pCO}^{2}=$ partial pressure of $\mathrm{CO}_{2}, \mathrm{BE}=$ base excess, $\mathrm{SB}=$ standard bicarbonate, $\mathrm{AST}=$ aspartate transaminase,

GMT = gama-glutamyl transferase 
Table 5. Results of tuber coxae cancellous tissue examination

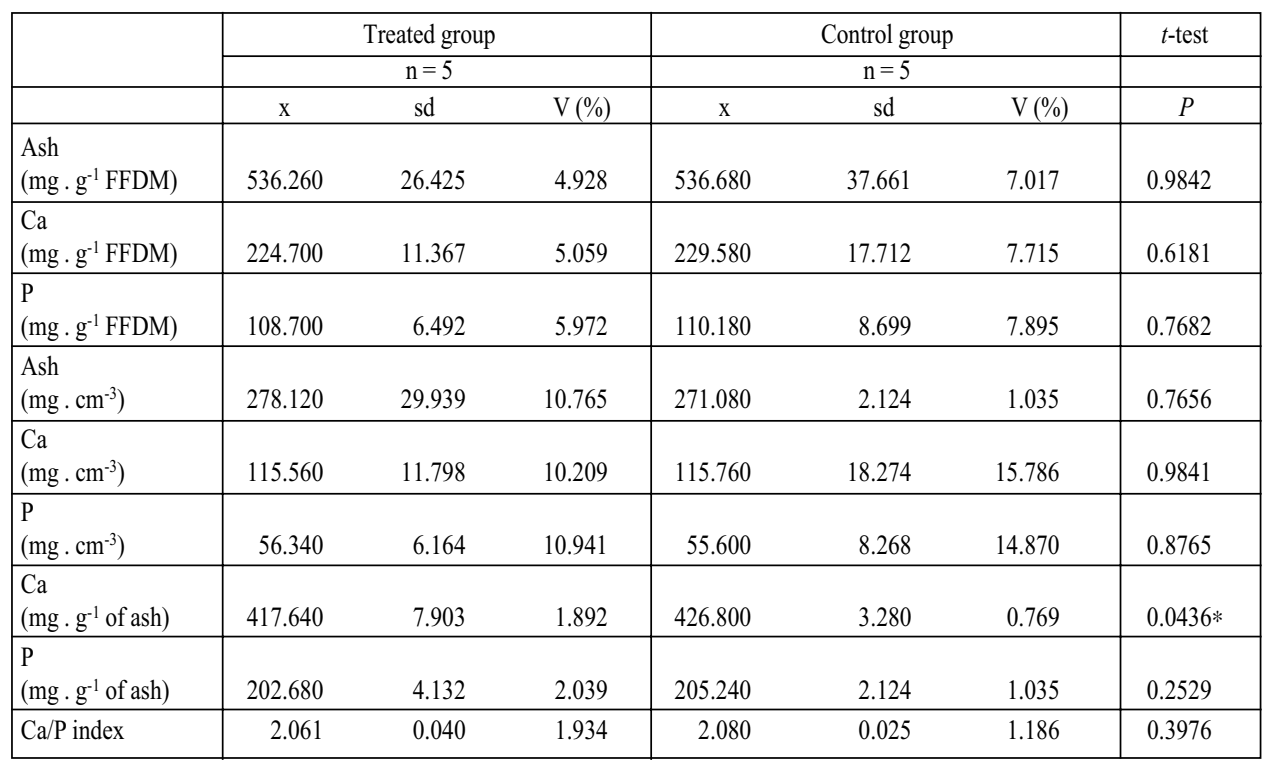

FFDM $=$ fat free dry matter

\section{Discussion}

The consumption of the high-grain diet by the bulls resulted in a significant decrease of $\mathrm{pH}$ and increase of total acidity in rumen fluid in the treated group, as compared with the control one, fed a maize silage-based diet. The results correspond with those received by many researchers who observed a decrease in rumen fluid $\mathrm{pH}$ when feeding cattle with high amounts of grain (Harmon et al. 1985; Leedle et al. 1995; Goad et al. 1998; Hristov et al. 2001; Schoon maker et al.2003). Unlike the results reported by the above-mentioned authors, the rumen fluid $\mathrm{pH}$ value in the high-grain diet group did not drop under 6.0, and the values outside the reference range reported by Jagoš and Bouda (1981) were found only in several animals. The high-grain diet group showed a significant decrease in infusorial counts. Similar results were reported by Hristov et al. (2001), who found markedly lower infusorial counts in animals fed diets with high proportions of concentrate than in those fed diets with less concentrate. There was no significant difference found between the groups in ammonia concentrations, except for the first rumen fluid sampling with the high-grain diet group showing a significantly higher value. This finding is in contradiction to the claims by Goad et al. (1998) and Hristov et al. (2001) who both reported a decrease in rumen fluid ammonia concentrations after a switch to a highly concentrated diet. Unlike the researchers who reported increased lactic acid levels due to feeding high-grain diets (Harmon et al. 1985; Leedle et al. 1995; Goad et al. 1998), we did not observe any significant differences between the groups (except for the first rumen fluid sampling). Lactate levels were within the reference range (Jagoš and B ou da 1981). Similar results were received by Hristov et al. (2001), who found low lactate levels in animals fed a diet containing 95\% concentrate. Due to the consumption of high levels of grain, the high-grain diet group showed a significant increase in total VFA levels in rumen fluid. Similar results were obtained by Harmon et al. (1985), Leedle et al. (1995) and Goad et al. (1998). Both Leedle et al. (1995) and Goad et al. (1998) reported that with 
an increasing concentrate proportion in the diet a percentage of acetate was decreasing and percentages of propionate, butyrate and valerate were increasing. Proportions of different VFAs found in the high-grain diet group correspond with the above-mentioned findings, except for butyrate. Unlike the results reported in the literature, the high-grain diet group showed significantly higher proportions of butyrate than the control group with the last two rumen fluid samplings.

Blood $\mathrm{pH}$ was decreased in both the groups, when compared with the reference values (Jagoš and Bouda 1981), and, on the other hand, $\mathrm{pCO}_{2}$ values were increased. Based on the results obtained it can be assumed that these values were not influenced by the type of diet. The $\mathrm{pCO}_{2}$ increase occurred probably due to difficult handling of animals during the blood sampling, which resulted also in the $\mathrm{pH}$ drop. Many researchers (Harmon et al. 1985; Goad et al. 1998; Hersom et al. 2003) found that blood pH values were not significantly influenced by high-grain diets. On the other hand, Leedle et al. (1995) observed a development of metabolic acidosis during a period of adaptation to a $90 \%$ concentrate diet. In spite of expectations, higher SB and BE values were found in the group receiving the high-grain diet. However, differences between the groups were not significant, except for the third sampling. SB and BE values showed a descending trend in both the groups. Particularly with the last sampling the mean values in both groups were markedly decreased, however, they did not drop under the lower reference range limit (Jag oš and Bouda 1981). Also Goad et al. (1998) observed a gradual decrease in bicarbonate concentrations and BE during the feeding of the high-grain diet. Changes in bicarbonate concentrations during the transfer to a high-grain diet were also noted by Harmon et al. (1985). On the contrary, Hersom et al. (2003) found that the feeding of a high-grain diet influenced neither bicarbonate concentrations nor BE.

For the evaluation of liver functions, serum levels of total bilirubin and liver enzymes AST and GMT were measured. Total bilirubin concentrations were lower in the treated group than in the control one at all the samplings, however, differences between the groups were not statistically significant. The AST activity was increased in both the groups with all the samplings, with the last sampling the treated group showed a significantly higher value. An increased AST activity was also observed by Lal et al. (1991) in goats administered cereal grain intraruminally. On the other hand, Brown et al. (1999) did not observe any effects of a diet with $90 \%$ concentrate on total bilirubin levels and AST activity in the serum of sheep. There were no significant differences found in the GMT activity between the groups. Some literature sources reported an increased occurrence of liver abscesses found after the slaughter (Nagaraja and Chengappa 1998; Galyean and Rivera 2002).

Although some authors (Patra et al. 1993; B rown et al. 2000) reported increased serum $\mathrm{Ca}$ level during rumen acidosis, the $\mathrm{Ca}$ concentrations we observed in the treated group were within the reference range (Jagoš and Bouda 1981) and differences between the groups were not significant. Serum P concentrations were increased in the treated group, however, the difference between the groups was significant only with the second sampling. Also Patra et al. (1993) and B rown et al. (1999) reported increased plasma P concentrations in sheep with experimentally induced acidosis. On the contrary, B rown et al. (2000) reported decreased serum $P$ levels in cattle after the intraruminal administration of cereal grains.

Ash content in $1 \mathrm{~g}$ FFDM of the tuber coxae cancellous tissue was found lower in both the experimental groups as compared with values found by Doubek et al. (1994) in clinically healthy bulls, but they were higher than the values these authors found in bulls with clinical osteopathies. Ash weight in $1 \mathrm{~cm}^{3}$ cancellous tissue in both the treated and control groups corresponded with values reported in healthy fattening bulls. $\mathrm{Ca} / \mathrm{P}$ index in ash in both the groups also corresponded with values found by Doubek et al. (1994) in clinically healthy 
bulls. The only effect of feeding the high-grain diet observed was that on Ca content in bone tissue, with the bulls fed the high-grain diet showing lower mean Ca levels in $1 \mathrm{~g}$ FFDM and $1 \mathrm{~g}$ ash than the control ones. Ca content in $1 \mathrm{~g}$ ash was significantly lower in the high-grain diet group; in other parameters under study no significant differences between the groups were found.

\section{Vliv intenzivního výkrmu býků na bázi zrnin na vybrané ukazatele zdravotního stavu}

Cílem práce bylo posoudit vliv intenzivního výkrmu býků s vysokým podílem zrnin na vybrané ukazatele zdravotního stavu. Sledované ukazatele metabolického profilu byly srovnány s výsledky při tradičním výkrmu na bázi kukuřičné siláže. Do pokusu bylo zařazeno 18 býků českého strakatého plemene, kterým se podávala krmná dávka obsahující 75,0 - 83,6 \% koncentrátů v sušině. V kontrolní skupině bylo použito 18 býkủ stejného plemene krmených směsnou krmnou dávkou na bázi kukuřičné siláže. Pokus byl zahájen od odstavu telat v průměrném věku 114 dnů u pokusné a 115 dnů u kontrolní skupiny a ukončen porážkou býků v průměrném věku 473 dnů u pokusné a 474 dnů u kontrolní skupiny. Délka trvání pokusu byla celkem 359 dnů.

Během výkrmu byly odebírány vzorky krve a bachorové tekutiny k posouzení bachorové fermentace, acidobazické rovnováhy a vybraných biochemických ukazatelů. Po porážce byly odebrány vzorky spongiózy tuber coxae k posouzení metabolismu kostí. Ze sledovaných ukazatelů byly výkrmem na bázi obilovin ovlivněny zejména ukazatele bachorové fermentace. Býci pokusné skupiny měli statisticky významně $(P \leq 0,05)$ nižší pH bachorové tekutiny a vyšší celkovou titrační aciditu, vysoce významně $(P \leq 0,01)$ vyšší obsah celkových TMK a podíl propionátu a vysoce významně $(P \leq 0,01)$ nižší podíl acetátu ve srovnání s kontrolní skupinou. Byl zde také vysoce významně $(P \leq 0,01)$ nižší počet nálevníků. Acidobazická rovnováha nebyla podle zjištěných výsledků výkrmem na bázi obilovin významně ovlivněna. Býci pokusné skupiny vykazovali dále zvýšené hodnoty AST a fosforu v krevním séru, rozdíly oproti kontrolní skupině však byly převážně statisticky neprůkazné. Ve vzorcích spongiózy tuber coxae byl u pokusné skupiny statisticky významně $(P \leq 0,05)$ nižší obsah vápníku v 1 g popela kostní tkáně, v dalších sledovaných ukazatelích nebyly statisticky průkazné rozdíly.

\section{Acknowledgements}

This study was supported by IGA VFU (Internal Grant Agency at the University of Veterinary and Pharmaceutical Sciences Brno), within the project No. 3/2004/FVHE.

\section{References}

ANONYMOUS: Notice No. 124/2001, Annex 50, Ministry of Agriculture of the Czech Republic (in Czech) BROWN MS, HALLFORD DM, GALYEAN ML, KREHBIEL CR, DUFF G 1999: Effect of ruminal glucose infusion on dry matter intake, urinary nitrogen composition, and serum metabolite and hormone profiles in ewes. J Anim Sci 77: 3068-3076

BROWN MS, KREHBIEL CR, GALYEAN ML, REMMENGA MD, PETERS JP, HIBBARD B, ROBINSON J, MOSELEY WM 2000: Evaluation of models of acute and subacute acidosis on dry matter intake, ruminal fermentation, blood chemistry, and endocrine profiles of beef steers. J Anim Sci 78: $3155-3168$

DOUBEK J, ILLEK J, ONDRÁČ́EK J 1994: Pre-clinical diagnosis of metabolic osteopathy in fattened bulls. Vet Med - Czech 39: 231-243 (in Czech)

GALYEAN ML, RIVERA JD 2002: Nutritionally related disorders affecting feedlot cattle. Can J Anim Sci 82: 13-20

GOAD DW, GOAD CL, NAGARAJA TG 1998: Ruminal microbial and fermentative changes associated with experimentally induced subacute acidosis in steers. J Anim Sci 76: 234-241

HARMON DL, BRITTON RA, PRIOR RL, STOCK RA 1985: Net portal absorption of lactate and volatile fatty acids in steers experiencing glucose-induced acidosis or fed a 70\% concentrate diet ad libitum. J Anim Sci 60: 560-569 
HERSOM MJ, KREHBIEL CR, HORN GW, KIRKPATRICK JG 2003: Effect of the live weight gain of steers during winter grazing on digestibility, acid-base balance, blood flow, and oxygen consumption by splanchnic tissues during adaptation and subsequent feeding of a high-grain diet. J Anim Sci 81: 3130-3140

HRISTOV AN, IVAN M, RODE LM, McALLISTER TA 2001: Fermentation characteristics and ruminal ciliate protozoal populations in cattle fed medium- or high-concentrate barley-based diets. J Anim Sci 79: 515-524

JAGOŠ P, BOUDA J 1981: Basic biochemical and physiological values in farm animals and novel formulation of laboratory examination results. ÚVO, Pardubice, 29 p. (in Czech)

LAL SB, SWARUP D, DWIVEDI SK, SHARMA MC 1991: Biochemical alterations in serum and cerebrospinal fluid in experimental acidosis in goats. Res Vet Sci 50: 208-210

LEEDLE JAZ, COE ML, FREY RA 1995: Evaluation of health and ruminal variables during adaptation to grainbased diets in beef-cattle. Am J Vet Res 56: 885-892

NAGARAJA TG, CHENGAPPA MM 1998: Liver abscesses in feedlot cattle: A review. J Anim Sci 76: 287-298

NOCEK JE 1997: Bovine acidocis: implications on laminitis. J Dairy Sci 80: 1005-1028

OWENS FN, SECRIST DS, HILL WJ, GILL DR 1998: Acidosis in cattle: A review. J Anim Sci 76: 275-286

PATRA RC, LAL SB, SWARUP D 1993: Physiochemical alteration in blood, cerebrospinal fluid, and urine in experimental lactic acidosis in sheep. Res Vet Sci 54: 217-220

SCHOONMAKER JP, CECAVA VM, FAULKNER DB, FLUHARTY FL, ZERBY HN, LOERCH SC 2003: Effect of source of energy and rate of growth on performance, carcass characteristics, ruminal fermentation, and serum glucose and insulin of early-weaned steers. J Anim Sci 81: 843-855

SOMMER A, ČEREŠÑÁKOVÁ Z, FRYDRYCH Z, KRÁLÍK O, KRÁLÍKOVÁ Z, KRÁSA A, PAJTÁS̆ M, PETRIKOVIČ P, POZDÍŠEK J, ŠIMEK M, TŘINÁCTÝ J, VENCL B, ZEMAN L 1994: Nutrient requirements and tables of nutrient value of ruminant feed. ČZS VÚVZ, Pohořelice, 198 p. (in Czech) 